\title{
Enhanced Secure SWIPT in Heterogeneous Network via Intelligent Reflecting Surface
}

\author{
Jie Yang $\mathbb{D}^{1},{ }^{1}$ Xinsheng Ji, ${ }^{1}$ Kaizhi Huang $\mathbb{D},{ }^{1}$ Xiaoli Sun, ${ }^{1}$ and Yi Wang ${ }^{2}$ \\ ${ }^{1}$ Information Engineering University, Zhengzhou 450002, China \\ ${ }^{2}$ Zhengzhou University of Aeronautics, Zhengzhou 450046, China \\ Correspondence should be addressed to Kaizhi Huang; huangkaizhi@tsinghua.org.cn
}

Received 30 September 2020; Revised 8 January 2021; Accepted 16 January 2021; Published 8 March 2021

Academic Editor: Petros Nicopolitidis

Copyright $(02021$ Jie Yang et al. This is an open access article distributed under the Creative Commons Attribution License, which permits unrestricted use, distribution, and reproduction in any medium, provided the original work is properly cited.

In this paper, secure transmission in a simultaneous wireless information and power transfer technology-enabled heterogeneous network with the aid of multiple IRSs is investigated. As a potential technology for $6 \mathrm{G}$, intelligent reflecting surface (IRS) brings more spatial degrees of freedom to enhance physical layer security. Our goal is to maximize the secrecy rate by carefully designing the transmit beamforming vector, artificial noise vector, and reflecting coefficients under the constraint of quality-of-service. The formulated problem is hard to solve due to the nonconcave objective function as well as the coupling variables and unit-modulus constraints. Fortunately, by using alternating optimization, successive convex approximation, and sequential Rank-1 constraint relaxation approach, the original problem is transformed into convex form and a suboptimal solution is achieved. Numerical results show that the proposed scheme outperforms other existing benchmark schemes without IRS and can maintain promising security performance as the number of terminals increases with lower energy consumption.

\section{Introduction}

Recently, with the vigorous development of the mobile Internet of Things (IoT), higher data rate and seamless network coverage will be required in the next generation network. The heterogeneous network (HetNet) with simultaneous wireless information and power transfer technology (SWIPT) provides a promising green solution for the connection requirements and power supply of massive energy-limited IoT nodes $[1,2]$.

However, SWIPT-enabled HetNet poses unprecedented security challenges to traditional security strategies. On the one hand, dynamic network topology makes it difficult for access control and key distribution management. On the other hand, energy receiver (ER) is closer to base stations (BSs) than information receiver (IR) and the behavior of energy harvesting and information decoding is uncontrollable by BS, which has the potential to become an eavesdropper [3]. In recent years, physical layer security (PLS) has emerged as a lightweight secure technique by utilizing the inherent characteristics of wireless environment [4]. Compared to conventional single-tier networks, the complicated multilayer network architectures with the co-frequency mutual interference and the unsubscribed ER make it more challenging to implement PLS. In [5], artificial noise (AN) scheme is exploited to degrade eavesdropping capability in a SWIPT-enabled two-tier HetNet and a near optimal solution based on successive convex approximation (SCA) for secrecy rate maximization is proposed. In [6], a more complex multitier HetNet where exist one macro base station (MBS) and multiple femto base stations (FBS) in co-channel deployment with the goal of sum logarithmic secrecy rate maximization is considered. Then, centralized and distributed secure beamforming schemes are discussed, respectively. Furthermore, in [7], the authors explore the robust secrecy energy efficiency maximization designs under deterministic channel state information (CSI) error, and a suboptimal algorithm based on alternative direction multiplier method is proposed. However, existing works have some drawbacks. In order to make the legitimate signal sent by BS lie into the null space of 
malicious ERs' channels, additional transmitting antennas are needed, which in turn increases the complexity of the equipment. In order to degrade the ERs' channels, the AN technique is adopted, which in turn consumes extra energy. This motivates us to consider a novel scheme to increase the spatial dimension with lower energy consumption.

Meanwhile, intelligent reflecting surface (IRS), which is a planar array consisting of a large number of low-cost composite material elements, has been regarded as a revolutionary technique to improve energy efficiency for 6G wireless networks [8]. Each element at the IRS can adjust the reflecting coefficients independently, and the combined signal composed of the direct-link and reflectlink signal at intended receivers can be enhanced or weakened. Moreover, due to passive characteristics, less power is consumed than traditional transceivers or relays [9]. Thus, as a new promising solution, IRS has been studied in different scenarios such as physical layer security $[10,11]$, coverage extension [12], energy efficiency enhancement [13], and so on. In [14], IRS-assisted secure communication in multiple-input multiple-output system with two different phase shifting capabilities is investigated. In [15], the authors focus on exploiting multiple IRSs in a SWIPT system for improving the wireless power transfer efficiency. In [16], the secure issue assisted with IRS is discussed in a SWIPT-enabled multiple-input single-output system, while this model does not consider ER as a potential eavesdropper.

To the best of our knowledge, by far there is no prior work that studies IRS enhanced secure communication problem for SWIPT-enabled HetNets. Massive passive elements at the IRS can provide more degrees of freedom (DoF) to strike a balance between secure energy efficiency and system complexity. Moreover, under the constraint of quality-of-service (QoS), complex cross-layer interference and more designing parameters pose challenges for resource allocation. Thus, how to jointly optimize the secure transmit precoders and the phase shifts is a meaningful but still an open problem.

Motivated by the aforementioned observations, in this paper, we study an IRS-assisted SWIPT HetNet system aimed at maximizing the secrecy rate of the IR, while guaranteeing QoS requirements of microusers (MUs) and ERs. An efficient solution based on alternating optimization and SCA technique is proposed. Our main contributions are summarized as follows:

(1) It is the first time to exploit IRSs to enhance security performance of SWIPT-enabled HetNet system, in which the IRSs are deployed nearby the MBS and FBS, respectively. FBS shares downlink spectrum resource with MBS. In this model, secure beamforming and $\mathrm{AN}$ techniques are utilized to ensure secure communication and charging. Meanwhile, the IRSs provide extra DoF to properly exploit mutual channel interference. Then, a resource allocation problem aimed at secrecy rate maximization is established by jointly designing the transmit precoder, artificial noise vector, and reflecting coefficients.

(2) A suboptimal iterative algorithm is proposed. The problem is highly nonconvex because of the strong coupling of the design variables. To tackle it, we first decompose the problem into two subproblems by applying an alternating optimization algorithm. Then, each subproblem is transformed into convex form step by step with SCA and semidefinite relaxation (SDR) techniques. Moreover, to ensure the convergence of the algorithm, for the first subproblem, the rank of the optimal solution is proved to be 1 , and for the second subproblem, sequential Rank-1 constraint relaxation (SROCR) approach is utilized to approximate Rank-1 solution. Finally, the computational complexity is analyzed.

(3) The simulation results show that by carefully designing the reflecting coefficients of IRSs, the secrecy performance can be significantly enhanced compared to conventional schemes without IRS. Moreover, when the number of low-cost reflecting units increases, the proposed transmission strategy can provide higher secrecy rate with low energy consumption, which further verifies that our scheme is quite suitable for future massive IoT node scenario.

The remainder of this paper is organized as follows. Section 2 introduces the system model and formulates the secrecy rate maximization problem under QoS constraints. Section 3 focuses on the secure beamforming design. Section 4 and Section 5 provide simulation results and conclusions, respectively.

\section{Signal Model and Problem Formulation}

2.1. Signal Model. An IRS-assisted SWIPT HetNet system is considered, where exist a macrocell and a femtocell, as depicted in Figure 1. The macrocell shares the same spectrum resources as the femtocell. The MBS equipped with $M_{a}$ antennas serves $M$ single-antenna MUs. The FBS equipped with $M_{b}$ antennas serves $K+1$ single-antenna FUs, one IR, and $K$ ERs. Based on the mechanism of [17], separate ER and IR are adopted. IRS-1 and IRS-2 are deployed on walls or buildings near MBS and FBS (as illustrated in [18], the large-scale loss of the reflection path and the product of the distances of the BS-IRS and IRS-user channels are in the direct ratio; when the IRS is deployed far away from users or BSs, the incremental performance is relatively small; considering the location uncertainty of the users in HetNet, deploying the IRS near the BSs is a relatively optimal choice), respectively, and are controlled by the BS via separate wireless link. Since FBS cannot strictly control the behavior of ERs, the malicious ER may change its mode to decode confidential information intended for the legitimate IRs. Further, we assume that the ERs can only wiretap the IR in the same femtocell, due to long-distance loss. Since the MU has a 


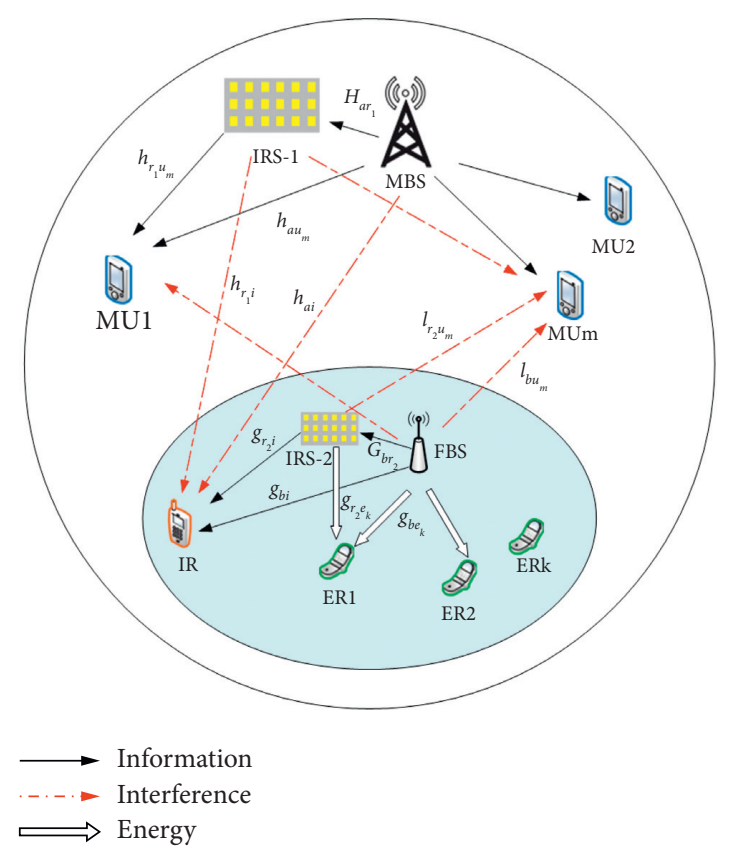

Figure 1: System model.

stronger ability against eavesdropping than the IR and its main consideration is high transmission rate, eavesdroppers are not considered in the macrocell. For notational simplicity, the $k$ th ER in the femtocell and the $m$ th $\mathrm{MU}$ in the macrocell are denoted by $\mathrm{ER}_{k}$ and $\mathrm{MU}_{m}$, respectively. The set of ERs is represented as $\mathscr{K}=$ $\{1, \ldots, K\}$, and the set of MUs is represented as $\mathscr{M}=\{1, \ldots, M\}$.

In this paper, a quasi static Rician fading environment is considered. The channel vectors from MBS to IRS-1, $\mathrm{MU}_{m}$, $\mathrm{IR}$, and $\mathrm{ER}_{k}$ are denoted by $\mathbf{H}_{a r_{1}}^{H} \in \mathbb{C}^{N_{1} \times M_{a}}, \mathbf{h}_{a u_{m}}^{H} \in \mathbb{C}^{1 \times M_{a}^{m}}$, $\mathbf{h}_{a i}^{H} \in \mathbb{C}^{1 \times M_{a}}$, and $\mathbf{h}_{a e_{k}}^{H} \in \mathbb{C}^{1 \times M_{a}}$, respectively. Similarly, the channel vectors from FBS to IRS-2, IR, and $\mathrm{ER}_{k}$ are denoted by $\mathbf{G}_{b r_{2}}^{H} \in \mathbb{C}^{N_{2} \times M_{b}}, \mathbf{g}_{b i}^{H} \in \mathbb{C}^{1 \times M_{b}}$, and $\mathbf{g}_{b e_{k}}^{H} \in \mathbb{C}^{1 \times M_{b}}$, respectively; the interference channel vector from FBS to $\mathrm{MU}_{m}$ is denoted by $\mathbf{l}_{b u_{m}}^{H} \in \mathbb{C}^{1 \times M_{b}}$; the reflecting channel vectors from IRS- 1 to $\mathrm{ER}_{k}, \mathrm{IR}$, and $\mathrm{MU}_{m}$ are represented as $\mathbf{h}_{r_{1}, e_{k}}^{H} \in \mathbb{C}^{1 \times N_{1}}$, $\mathbf{h}_{r_{1} i}^{H} \in \mathbb{C}^{1 \times N_{1}}$, and $\mathbf{h}_{r_{1} u_{m}}^{H} \in \mathbb{C}^{1 \times N_{1}}$, respectively; the channel vectors from IRS-2 to $\mathrm{ER}_{k}$, IR, and $\mathrm{MU}_{m}$ are represented as $\mathbf{g}_{r_{2} e_{k}}^{H} \in \mathbb{C}^{1 \times N_{2}}, \mathbf{g}_{r_{2} i}^{H} \in \mathbb{C}^{1 \times N_{2}}$, and $l_{r_{2} u_{m}}^{H} \in \mathbb{C}^{1 \times N_{2}}$, respectively. Then, the phase shift matrix at the IRS is represented as $\Phi_{j}=\operatorname{diag}\left(e^{j \theta_{1}}, e^{j \theta_{2}}, \ldots, e^{j \theta_{N_{j}}}\right), j=1,2$, where $\theta_{n} \in[0,2 \pi]$ is the phase shift on the incident signal at its $n$th reflecting element. Notice that passive eavesdropper's CSI is hard to obtain, while the ER, as a user in the SWIPT network, needs to feed back information to the FBS [17]; it is reasonable that ERs' CSI can be acquired by the FBS. And in this paper, centralized manner is adopted for HetNet. Global CSI of all channel vectors is supposed to be known by MBS and FBS, where local CSI can be shared via wired link between MBS and FBS. From Figure 1, the signal obtained at each user is composed of reflected signal and direct signal which is different from the model without IRS and thus needs to be reanalyzed.

The transmitted signal from MBS is given by

$$
\mathbf{x}_{\mathrm{MBS}}=\sum_{i=1}^{M} \mathbf{w}_{m} s_{m},
$$

where $s_{m} \sim C N(0,1)$ intended for $\mathrm{MU}_{m}$ is the information signal transmitted by MBS and $\mathbf{w}_{m}$ is the associated beamforming vector.

Considering the presence of malicious ERs in the femtocell, a secure beamforming scheme with the aid of AN is exploited. Thus, the signal sent by FBS is expressed as

$$
\mathbf{x}_{\mathrm{FBS}}=\mathbf{w}_{I} s_{I}+\mathbf{f} a,
$$

where $s_{I} \sim C N(0,1)$ and $a \sim C N(0,1)$ represent the independent information signal and AN signal and $\mathbf{w}_{I}$ and $\mathbf{f}$ represent the corresponding beamforming and AN vectors, respectively.

The signal received at $\mathrm{MU}_{m}$ contains not only the target signal sent by MBS but also the co-channel interference from FBS and the reflecting signal from IRS-1 or IRS-2, and thus the signal can be given as 


$$
y_{m}=\underbrace{\left(\mathbf{h}_{a u_{m}}^{H}+\mathbf{h}_{r_{1} u_{m}}^{H} \Phi_{1} \mathbf{H}_{a r_{1}}\right) \mathbf{w}_{m} s_{m}}_{\text {target signal }}+\underbrace{\sum_{j=1, j \neq m}^{M}\left(\mathbf{h}_{a u_{m}}^{H}+\mathbf{h}_{r_{1} u_{m}}^{H} \Phi_{1} \mathbf{H}_{a r_{1}}\right) \mathbf{w}_{j} s_{j}}_{\text {intra-layer interference }}+\underbrace{\left(\mathbf{l}_{b u_{m}}^{H}+\mathbf{l}_{r_{2} u_{m}}^{H} \Phi_{2} \mathbf{G}_{b r_{2}}\right) \mathbf{x}_{\mathrm{FBS}}}_{\text {inter-layer interference }}+\underbrace{n_{m}}_{\text {noise }},
$$

where $n_{m} \sim C N\left(0, \sigma_{m}^{2}\right)$ is the additive white Gaussian noise (AWGN) with variance $\sigma_{m}^{2}$ at $\mathrm{MU}_{m}$.

Let $v_{j}^{H}=\left[v_{1}, v_{2}, \ldots, v_{N_{j}}\right]$, where $v_{n}=e^{j \theta_{n}}, n=1,2, \ldots$, $N_{j}, j=1,2$. By changing variables as $\mathbf{h}_{r_{1} u_{m}}^{H} \Phi_{1} \mathbf{H}_{a r_{1}}=$
$\mathbf{v}_{1}^{H} \mathbf{H}_{a r_{1} u_{m}}, \quad \mathbf{l}_{r_{2} u_{m}}^{H} \Phi_{2} \mathbf{G}_{b r_{2}}=\mathbf{v}_{2}^{H} \mathbf{L}_{b r_{2} u_{m}}$, where $\quad \mathbf{H}_{a r_{1} u_{m}}=\operatorname{diag}$ $\left(\mathbf{h}_{r_{1} u_{m}}^{H}\right) \mathbf{H}_{a r_{1}}, \mathbf{L}_{b r_{2} u_{m}}=\operatorname{diag}\left(\mathbf{l}_{r_{2} u_{m}}^{H}\right) \mathbf{G}_{b r_{2}}$, we can obtain the signal-to-interference-plus noise ratio (SINR) at $\mathrm{MU}_{m}$ as

$$
\operatorname{SINR}_{m}=\left|\widetilde{\mathbf{v}}_{1}^{H} \widetilde{H}_{a u_{m}} \mathbf{w}_{m}\right|^{2} \times\left\{\frac{\left|\widetilde{v}_{1}^{H} \widetilde{H}_{a u_{m}} \mathbf{w}_{m}\right|^{2}}{\sum_{j=1, j \neq m}^{M}\left|\widetilde{v}_{1}^{H} \widetilde{H}_{a u_{m}} \mathbf{w}_{j}\right|^{2}+\left|\widetilde{v}_{2}^{H} \widetilde{L}_{b u_{m}} \mathbf{w}_{I}\right|^{2}+\left|\widetilde{v}_{2}^{H} \widetilde{L}_{b u_{m}} \mathbf{f}\right|^{2}+\sigma_{m}^{2}}\right\}^{-1},
$$

where $\widetilde{H}_{a u_{m}}=\left[\begin{array}{c}\mathbf{H}_{a r_{1} u_{m}} \\ \mathbf{h}_{a u_{m}}^{H}\end{array}\right], \widetilde{L}_{b u_{m}}=\left[\begin{array}{c}\mathbf{L}_{b r_{2} u_{m}} \\ \mathbf{l}_{b u_{m}}^{H}\end{array}\right], \widetilde{v}_{2}^{H}=e^{j \bar{w}}\left[\mathbf{v}_{2}^{H}, 1\right]$, and $\bar{w}$ is a slack variable and is set as an arbitrary phase rotation.
Similarly, considering the co-channel interference, the signal received at IR can be given by

$$
y_{I}=\underbrace{\left(\mathbf{g}_{b i}^{H}+\mathbf{g}_{r_{2} i}^{H} \Phi_{2} \mathbf{H}_{b r_{2}}\right) \mathbf{w}_{I} \boldsymbol{s}_{I}}_{\text {target signal }}+\underbrace{\left(\mathbf{g}_{b i}^{H}+\mathbf{g}_{r_{2} i}^{H} \Phi_{2} \mathbf{H}_{b r_{2}}\right) \mathbf{f} a}_{\text {intra-layer interference }}+\underbrace{\sum_{m=1}^{M}\left(\mathbf{h}_{a i}^{H}+\mathbf{h}_{r_{1} i}^{H} \Phi_{1} \mathbf{H}_{a r_{1}}\right) \mathbf{w}_{m} \boldsymbol{s}_{m}}_{\text {inter-layer interference }}+\underbrace{n_{I}}_{\text {noise }},
$$

where $n_{I} \sim C N\left(0, \sigma_{I}^{2}\right)$ denotes the AWGN at IR. Like $\mathrm{MU}_{m}$, the corresponding SINR at IR can be derived as

$$
\operatorname{SINR}_{I}=\frac{\left|\widetilde{v}_{2}^{H} \widetilde{G}_{b i} \mathbf{w}_{I}\right|^{2}}{\sum_{m=1}^{M}\left|\widetilde{v}_{1}^{H} \widetilde{H}_{a i} \mathbf{w}_{m}\right|^{2}+\left|\widetilde{v}_{2}^{H} \widetilde{G}_{b i} \mathbf{f}\right|^{2}+\sigma_{I}^{2}}
$$

where $\widetilde{G}_{b i}=\left[\begin{array}{c}\mathbf{G}_{b r_{2} i} \\ \mathbf{g}_{b i}^{H}\end{array}\right], \widetilde{\mathbf{H}}_{a i}=\left[\begin{array}{c}\mathbf{H}_{a r_{1} i} \\ \mathbf{h}_{a i}^{H}\end{array}\right], \mathbf{G}_{b r_{2} i}=\operatorname{diag}\left(\mathbf{g}_{r_{2} i}^{H}\right) \mathbf{G}_{b r_{2}}$, and $\mathbf{H}_{a r_{1} i}=\operatorname{diag}\left(\mathbf{h}_{r_{1} i}^{H}\right) \mathbf{H}_{a r_{1}}$.

Due to the openness of wireless environment, ERs can receive the information-bearing signal $\mathbf{x}_{\mathrm{FBS}}$ intended for IR. And malicious ER may change its mode to extract the secure information transformed to the IR rather than just receiving the energy. Thus, like IR, the SINR at malicious $\mathrm{ER}_{k}$ can be given by

$$
\operatorname{SINR}_{e_{k}}=\frac{\left|\widetilde{v}_{2}^{H} \widetilde{G}_{b e_{k}} \mathbf{w}_{I}\right|^{2}}{\sum_{m=1}^{M}\left|\widetilde{v}_{1}^{H} \widetilde{H}_{a e_{k}} \mathbf{w}_{m}\right|^{2}+\left|\widetilde{v}_{2}^{H} \widetilde{G}_{b e_{k}} \mathbf{f}\right|^{2}+\sigma_{e_{k}}^{2}}, \quad \forall k \in \mathscr{K},
$$

where $\quad \widetilde{G}_{b e_{k}}=\left[\begin{array}{c}\mathbf{G}_{b r_{2} e_{k}} \\ \mathbf{g}_{b e_{k}}^{H}\end{array}\right], \quad \widetilde{H}_{a e_{k}}=\left[\begin{array}{c}\mathbf{H}_{a r_{1} e_{k}} \\ \mathbf{h}_{a e_{k}}^{H}\end{array}\right], \quad \mathbf{G}_{b r_{2} e_{k}}=$ $\operatorname{diag}\left(\mathbf{g}_{r_{2} e_{k}}^{H}\right) \mathbf{G}_{b r_{2}}$, and $\mathbf{H}_{a r_{1} e_{k}}=\operatorname{diag}\left(\mathbf{h}_{r_{1} e_{k}}^{H}\right) \mathbf{H}_{a r_{1}}$.
Then, the total power usage of the whole system can be expressed as

$$
P_{\text {tot }}=\sum_{m=1}^{M}\left\|\mathbf{w}_{m}\right\|^{2}+\left\|\mathbf{w}_{I}\right\|^{2}+\|\mathbf{f}\|^{2}
$$

For $\mathrm{ER}_{k}$, when working on energy harvesting mode and considering the linear energy harvesting model [19], the harvested energy is expressed as

$$
E_{k}=\xi\left(\left|\widetilde{v}_{2}^{H} \widetilde{G}_{b e_{k}} \mathbf{w}_{I}\right|^{2}+\left|\widetilde{v}_{2}^{H} \widetilde{G}_{b e_{k}} \mathbf{f}\right|^{2}+\sigma_{e, k}^{2}\right), \quad \forall k \in \mathscr{K},
$$

where $\xi \in(0,1]$ represents the efficiency of energy conversion.

Due to the limited hardware capabilities, it is difficult to adopt a collaborative eavesdropping strategy for ERs, although they are arbitrarily distributed nearby FBS. Therefore, considering the noncolluding eavesdropping [20], the achievable instantaneous secrecy rate is written as

$$
R_{\mathrm{sec}}=\left[\log _{2}\left(1+\mathrm{SINR}_{I}\right)-\max _{k \in \mathscr{K}} \log _{2}\left(1+\operatorname{SINR}_{e, k}\right)\right]_{+},
$$

where $[x]^{+}=\max \{x, 0\}$ means the max of $x$ and 0 . 
2.2. Problem Formulation. From formulas (4)-(9), we can see that co-channel interference and $\mathrm{AN}$ vector may degrade the data rate of legitimate users, while it is a beneficial factor to harvest energy and weaken eavesdropping channel. Thus, it is very appealing to carefully design transmit precoder and phase shifts to cripple the eavesdropping capabilities at ERs and have a minimal effect at $\mathrm{MU}_{m}$ and IR. Based on the above analysis, our goal is to design the vector $\left\{\mathbf{w}_{m}\right\}, \mathbf{w}_{I}, \mathbf{f}$, $\widetilde{v}_{1}^{H}, \widetilde{v}_{2}^{H}$ to maximize the secrecy rate at IR, while subjecting to the energy requirements for each ER, SINR requirement for each $\mathrm{MU}$, and the total power usage. Accordingly, we can formulate the above problem as

$$
\begin{aligned}
& \max _{\left\{\mathbf{w}_{m}\right\}, \mathbf{w}_{I}, \mathbf{f}, \widetilde{v}_{1}, \widetilde{v}_{2}} R_{\mathrm{sec}}, \\
& \text { s.t. } \quad \operatorname{SINR}_{m} \geq \Gamma_{m}, \quad \forall m \in M, \\
& P_{\text {tot }} \leq P_{\text {th }}, \\
& E_{k} \geq Q_{k}, \quad \forall k \in \mathscr{K}, \\
& \left|v_{j, n}\right|=1, \quad n=1,2, \ldots, N_{j}, j=1,2,
\end{aligned}
$$

where $\Gamma_{m}$ denotes the required SINR at $\mathrm{MU}_{m}, P_{t h}$ represents the maximum power constraint, and $Q_{k}$ is the energy requirement at $\mathrm{ER}_{k}$, respectively. In addition, constraint (11c), which discusses the total transmit power constraint, can be easily extended to the separate power threshold at MBS and FBS. It is worth noting that due to the nonconcave objective function as well as the coupling variables and unit-modulus constraints, problems (11a)-(11e) are nonconvex, which are hard to solve optimally. In the following sections, we aim at developing an efficient algorithm to find a near optimal solution.

\section{Secure Beamforming Design}

In this section, firstly, alternating optimization is exploited to decompose the joint optimization problem into two subproblems. Then, for each subproblem, by using SDR and SCA-based iterative, nonconvex objective functions and constraints are approximated to convex form, which can be efficiently solved.

3.1. Optimizing $\left(\left\{\mathbf{w}_{m}\right\}, \mathbf{w}_{I}, \mathbf{f}\right)$ for Given $\left(\widetilde{v}_{1}, \widetilde{v}_{2}\right)$. For given $\left(\widetilde{v}_{1}, \widetilde{v}_{2}\right)$, we denote $\widehat{G}_{b j}=G_{b j} G_{b j}^{H}, \widehat{H}_{a j}=H_{a j} H_{a j}^{H}, j \in\left\{u_{m}\right.$, $\left.i, e_{k}\right\}$, and $\widehat{L}_{b u_{m}}=L_{b u_{m}} L_{b u_{m}}^{H}$, where $G_{b j}=\widetilde{v}_{2}^{H} \widetilde{G}_{b j}, H_{a j}=\widetilde{v}_{1}^{H}$ $\widetilde{H}_{a j}$, and $L_{b u_{m}}=\widetilde{v}_{2}^{H} \widetilde{L}_{b u_{m}}$ can be viewed as the equivalent channels from MBS/FBS to users, respectively. Define new matrices as $\mathbf{F}=\mathbf{f} \mathbf{f}^{H}, \mathbf{W}_{I}=\mathbf{w}_{I} \mathbf{w}_{I}^{H}$, and $\mathbf{W}_{m}=\mathbf{w}_{m} \mathbf{w}_{m}^{H}$. Then, it follows that $\mathbf{W}_{I}>0, \mathbf{W}_{m}>0, \mathbf{F}>0$, and $\operatorname{Rank}\left(\mathbf{W}_{I}\right)=$ $\operatorname{Rank}\left(\mathbf{W}_{m}\right)=\operatorname{Rank}(\mathbf{F})=1$. Due to Rank-1 constraints, the problem is intractable and the SDR technique is utilized to drop these constraints [21]. Then, exploiting the properties of matrix traces and introducing several new real-valued auxiliary variables $z, \varphi, v_{k}$, and $u$, we rewrite problems (11a)-(11e) equivalently as

$$
\begin{gathered}
\max _{\left\{\mathbf{W}_{\mathbf{m}}\right\}, \mathbf{W}_{\mathbf{I}}, \mathbf{F}} z, \\
\varphi \log _{2}\left(1+\frac{\operatorname{Tr}\left(\widehat{G}_{b i}^{H} \mathbf{W}_{I}\right)}{\sum_{m} \operatorname{Tr}\left(\widehat{H}_{a i}^{H} \mathbf{W}_{m}\right)+\operatorname{Tr}\left(\widehat{G}_{b i}^{H} \mathbf{F}\right)+\sigma_{I}^{2}}\right), \\
1+\frac{\operatorname{Tr}\left(\widehat{G}_{b e_{k}}^{H} \mathbf{W}_{I}\right)}{\log _{2}\left(v_{k}\right) \leq u, \quad \forall k \in \mathscr{K},} \\
\operatorname{Tr}\left(\widehat{H}_{a e_{k}}^{H} \mathbf{W}_{m}\right)+\operatorname{Tr}\left(\widehat{G}_{b e_{k}}^{H} \mathbf{F}\right)+\sigma_{e, k}^{2} \leq v_{k}, \\
\forall k \in \mathscr{K},
\end{gathered}
$$

$$
\frac{\operatorname{Tr}\left(\widehat{\mathbf{H}}_{a u_{m}}^{H} \mathbf{W}_{m}\right)}{\sum_{i=1, i \neq m}^{M} \operatorname{Tr}\left(\widehat{H}_{a u_{m}}^{H} \mathbf{W}_{i}\right)+\operatorname{Tr}\left(\widehat{L}_{b u_{m}}^{H}\left(W_{I}+F\right)\right)+\sigma_{m}^{2}} \geq \Gamma_{m},
$$

$\forall m \in \mathscr{M}$,

$$
\xi\left(\operatorname{Tr}\left(\widehat{G}_{b e_{k}}^{H} \mathbf{W}_{I}\right)+\operatorname{Tr}\left(\widehat{G}_{b e_{k}}^{H} \mathbf{F}\right)+\sum_{m} \operatorname{Tr}\left(\widehat{H}_{a e_{k}}^{H} \mathbf{W}_{m}\right) \geq Q_{k}\right),
$$

$\forall k \in \mathscr{K}$,

$$
\begin{aligned}
& \sum_{m=1}^{M} \operatorname{Tr}\left(\mathbf{W}_{m}\right)+\operatorname{Tr}\left(\mathbf{W}_{I}\right)+\operatorname{Tr}(\mathbf{F}) \leq P_{\mathrm{th}}, \\
& \mathbf{W}_{I} \underset{\succ}{0,} \mathbf{W}_{m} \underset{\succ}{0,} \mathbf{F} \underset{\succ}{0} .
\end{aligned}
$$

Note that problems (11a)-(11e) and problems (12a)-(12h) are equivalent due to the fact that formulas (12a)-(12d) hold with equality at the optimal solution. Then, it can be observed that since constraints (12c), $(12 \mathrm{~d})$, and (12e) are nonconvex, problems (12a)-(12h) remain nonconvex. To deal with this predicament, an iterative algorithm is utilized to approximate the original problem.

Transformation of Constraints (12d) and (12e) For the fractional and logarithmic forms in formulas (12d) and (12e), by introducing auxiliary variables $\left\{x_{3, k}, k \in \mathscr{K}\right\}$ and 
$\left\{x_{4, k}, k \in \mathscr{K}\right\}$ and using the properties of exponential operations, it can be derived into

$$
\sum_{m} \operatorname{Tr}\left(\widehat{H}_{a e_{k}}^{H} \mathbf{W}_{m}\right)+\operatorname{Tr}\left(\widehat{G}_{b e_{k}}^{H} \mathbf{F}\right)+\sigma_{e, k}^{2}+\operatorname{Tr}\left(\widehat{G}_{b e_{k}}^{H} \mathbf{W}_{I}\right) \leq 2^{x_{3, k}},
$$

$$
\begin{gathered}
\sum_{m} \operatorname{Tr}\left(\widehat{H}_{a e_{k}}^{H} \mathbf{W}_{m}\right)+\operatorname{Tr}\left(\widehat{G}_{b e_{k}}^{H} \mathbf{F}\right)+\sigma_{e, k}^{2} \geq 2^{x_{4, k}}, \\
x_{3, k}-x_{4, k} \leq u, \quad \forall k \in \mathscr{K} .
\end{gathered}
$$

Clearly, (13b) and (13c) are convex constraints while (13a) remains nonconvex following from the fact that both sides of the constraints are convex. To tackle it, first-order Taylor expansion is employed to approximate the exponential function of the right-hand side (13a) in an iterative manner. At the $n$th iteration, $x_{3, k}[n]$ is denoted as the value solved at the current iteration and $\bar{x}_{3, k}[n]$ is denoted as a feasible point. Then, as $\bar{x}_{3, k}[n] \longrightarrow x_{3, k}[n]$, the lower bound of the exponential function $2^{x_{3, k}}$ can be given by [22]

$$
2^{x_{3, k}} \geq 2^{\bar{x}_{3, k}[n]}\left[1+\left(x_{3, k}[n]-\bar{x}_{3, k}[n]\right) \ln 2\right], \quad \forall k \in \mathscr{K}
$$

With (14), (13a) can be expressed as the following linear inequality in an iterative manner:

$$
\begin{array}{r}
\sum_{m} \operatorname{Tr}\left(\widehat{H}_{a e_{k}}^{H} W_{m}\right)+\operatorname{Tr}\left(\widehat{G}_{b e_{k}}^{H} \mathbf{F}\right)+\sigma_{e, k}^{2}+\operatorname{Tr}\left(\widehat{G}_{b e_{k}}^{H} \mathbf{W}_{I}\right) \\
\leq 2^{\bar{x}_{3, k}[n]}\left[1+\left(x_{3, k}[n]-\bar{x}_{3, k}[n] \ln 2\right)\right], \quad \forall k \in \mathscr{K} .
\end{array}
$$

Furthermore, note that although some nonlinear solvers (e.g., Fmincon) can be utilized to solve the exponential inequality in (13b), the required computation time is high in general. Fortunately, based on the result in [23], by introducing slack variables $\tau_{j}, \forall j \in\{0,1, \ldots, q+3\}$, a series of conic constraints can be used to approximate formula (13b):

$$
\begin{aligned}
\tau_{0} & \leq \gamma_{1}, \\
\left\|\left[2+\frac{x_{4, k}}{2^{q-1}}, 1-\tau_{1}\right]\right\|_{2} & \leq 1+\tau_{1}, \\
\left\|\left[\frac{5}{3}+\frac{x_{4, k}}{2^{q}}, 1-\tau_{2}\right]\right\|_{2} & \leq 1+\tau_{2}, \\
\left\|\left[2 \tau_{1}, 1-\tau_{3}\right]\right\|_{2} & \leq 1+\tau_{3}, \\
\tau_{2}+\frac{\tau_{3}}{24}+\frac{19}{72} & \leq \tau_{4}, \\
\left\|\left[2 \tau_{j-1}, 1-\tau_{j}\right]\right\|_{2} & \leq 1+\tau_{j}, \quad j \in\{5, \ldots, q+3\},
\end{aligned}
$$

$$
\left\|\left[2 \tau_{q+3}, 1-\tau_{0}\right]\right\|_{2} \leq 1+\tau_{0}
$$

where $\gamma_{1}=\sum_{m} \operatorname{Tr}\left(\widehat{H}_{a e_{k}}^{H} \mathbf{W}_{m}\right)+\operatorname{Tr}\left(\widehat{G}_{b e_{k}}^{H} \mathbf{F}\right)+\sigma_{e, k}^{2}$, and the factor $q$ decides the accuracy of the approximation.

Transformation of Constraint (12c) For the fractional and logarithmic forms in formula (12c), we introduce auxiliary variables $x_{1}, x_{2}$, and after several exponential operations, we have

$$
\begin{array}{r}
\sum_{m} \operatorname{Tr}\left(\widehat{H}_{a i}^{H} W_{m}\right)+\operatorname{Tr}\left(\widehat{G}_{b i}^{H} F\right)+\sigma_{I}^{2} \leq 2^{x_{1}}, \\
\operatorname{Tr}\left(\widehat{G}_{b i}^{H} \mathbf{W}_{I}\right)+\sum_{m} \operatorname{Tr}\left(\widehat{H}_{a i}^{H} \mathbf{W}_{m}\right)+\operatorname{Tr}\left(\widehat{G}_{b i}^{H} \mathbf{F}\right)+\sigma_{I}^{2} \geq 2^{x_{2}}, \\
x_{1}-x_{2} \geq \varphi .
\end{array}
$$

Clearly, (17b) and (17c) are convex constraints while (17a) remains nonconvex. Next, we focus on nonconvex constraint (17a). Similar to (13a), for the $n$th SCA iteration, $x_{1}[n]$ is denoted as the value solved at the current iteration and $\bar{x}_{1}[n]$ is denoted as a feasible point; then, we can transform (17a) into convex form:

$$
\begin{aligned}
& \sum_{m} \operatorname{Tr}\left(\widehat{H}_{a i}^{H} \mathbf{W}_{m}\right)+\operatorname{Tr}\left(\widehat{G}_{b i}^{H} \mathbf{F}\right)+\sigma_{I}^{2} \\
& \leq 2^{\bar{x}_{1}[n]}\left[1+\left(x_{1}[n]-\bar{x}_{1}[n]\right) \ln 2\right] .
\end{aligned}
$$

For (17b), to reduce the computational complexity, like (13b), a series of conic constraints can be utilized to approximate the exponential cone. The details are omitted, due to space limitation.

Finally, the approximated form of problems (12a)-(12h) is given by problem (19). And we can easily observe that problem (19) is a convex semidefinite programming problem, which can be directly solved via the CVX solver. The detailed procedure is listed in Algorithm 1.

$$
\begin{array}{ll}
\max _{\left\{\mathbf{w}_{\mathrm{m}}\right\},} z, & \mathbf{w}_{\mathrm{I}}, \mathbf{F} \\
\text { s.t. } \quad(12 b),(12 f),(12 g),(12 h),(12 i) \\
\quad(13 c),(15),(16 a-16 g),(17 c),(18) .
\end{array}
$$

Notice that since the Rank-1 constraints are relaxed in (12a)-(12h), there is no guarantee that the rank of the obtained $\mathbf{W}_{I}^{*},\left\{\mathbf{W}_{m}^{*}\right\}$, and $\mathbf{F}^{*}$ is one and the vectors $\left\{\mathbf{w}_{m}\right\}$, $\mathbf{w}_{I}$, and $\mathbf{f}$ may not be directly obtained. If the obtained solution is not Rank-1, Gaussian randomization is used for recovering vector approximately [24]. Fortunately, for problems (12a)-(12h), we can obtain Proposition 1 and then directly recover $\left(\mathbf{w}_{I}, \mathbf{f}\right)$ based on the eigenvalue decomposition. Moreover, simulations show that the obtained $\mathbf{W}_{m}^{*}$ is always Rank-1, which indicates that the rank relaxation is tight. 
Initialization: set $n=0$ and initialize feasible solutions $\left(\bar{x}_{1}[0], \bar{x}_{3, k}[0]\right)$ to problem (19).

(1) Repeat: $n$; $=n+1$;

(2) Solve the convex problem (19) with $\left(\bar{x}_{1}[n-1], \bar{x}_{3, k}[n-1]\right)$ and the optimal values are denoted by $\left(x_{1}^{*}, x_{3, k}^{*}\right)$;

(3) Updata $\left(\bar{x}_{1}[n], \bar{x}_{3, k}[n]\right)=\left(x_{1}^{*}, x_{3, k}^{*}\right)$

(4) Until: convergence

Algorithm 1: SCA-based iterative algorithm for solving problem (19).

Proposition 1. The solution $\mathbf{W}_{I}^{*}, \mathbf{F}^{*}$ solved in problems (12a)-(12h) must be Rank-1.

Proof. See Appendix A.

3.2. Optimizing $\left(w_{m}, w_{I}, f\right)$ for Given $\left(\left\{\mathbf{w}_{m}\right\}, \mathbf{w}_{I}, \mathbf{f}\right)$. For given $\left(\widetilde{V}_{1}, \widetilde{V}_{2}\right)$, we denote $\bar{G}_{b j}=G_{b j} G_{b j}^{H}, \bar{G}_{b j f}=G_{b j f} G_{b j f}^{H}$, $\bar{H}_{a j m}=H_{a j m} H_{a j m}^{H}, j \in\left\{u_{m}, i, e_{k}\right\}, \bar{L}_{b u_{m}}=L_{b u_{m}} L_{b u_{m}}^{H}$, and $\bar{L}_{b u_{m} f}$ $=L_{b u_{m} f} L_{b u_{m} f}^{H}$, where $G_{b j}=\widetilde{G}_{b j} \mathbf{w}_{I}, G_{b j f}=\widetilde{G}_{b j f} f, H_{a j m}=$ $\widetilde{H}_{a j} \mathbf{w}_{m}, L_{b u_{m}}=\widetilde{L}_{b u_{m}} \mathbf{w}_{I}$, and $L_{a j f}=\widetilde{L}_{a j f} \mathbf{f}$. Define new matrices as $\widehat{V}_{1}^{m}=\widehat{v}_{1} \widehat{v}_{1}^{H}, \widehat{V}_{2}=\widehat{v}_{2} \hat{v}_{2}^{H}$. Then, it follows that $\widehat{V}_{1} \underset{\succ}{0}$, $\widehat{V}_{2} 0$, and $\operatorname{Rank}\left(\widehat{V}_{1}\right)=\operatorname{Rank}\left(\widehat{V}_{2}\right)=1$. Besides, the diagonal elements of the matrix $\widehat{V}_{1}$ or $\widehat{V}_{2}$ are all 1 due to constraint (11e). Similar to (12a)-(12h), exploiting the properties of matrix traces and introducing several new real-valued auxiliary variables $z^{\prime}, \varphi^{\prime}$, and $u^{\prime}$, we rewrite problems (11a)-(11e) equivalently as

$$
\begin{array}{ll}
\max _{\widehat{V}_{1}, \widehat{V}_{2}} & z^{\prime}, \\
\text { s.t. } & z^{\prime} \leq \varphi^{\prime}-u^{\prime}, \\
& \varphi^{\prime} \leq \log _{2}\left(1+\frac{\operatorname{Tr}\left(\bar{G}_{b i}^{H} \widehat{V}_{2}\right)}{\sum_{m} \operatorname{Tr}\left(\bar{H}_{\text {aim }}^{H} \widehat{V}_{1}\right)+\operatorname{Tr}\left(\bar{G}_{b i f}^{H} \widehat{V}_{2}\right)+\sigma_{I}^{2}}\right),
\end{array}
$$

$\log _{2}\left(v_{k}\right) \leq u^{\prime}, \quad \forall k \in \mathscr{K}$,

$1+\frac{\operatorname{Tr}\left(\bar{G}_{b e_{k}}^{H} \widehat{V}_{2}\right)}{\sum_{m} \operatorname{Tr}\left(\bar{H}_{\mathrm{ame}_{k}}^{H} \bar{V}_{1}\right)+\operatorname{Tr}\left(\bar{G}_{b e_{k} f}^{H} \widehat{V}_{1}\right)+\sigma_{e, k}^{2}} \leq v_{k}, \quad \forall k \in \mathscr{K}$,

$$
\begin{aligned}
& \operatorname{Tr}\left(\bar{H}_{a u_{m} m}^{H} \widehat{V}_{1}\right) \sum_{j=1, j \neq m}^{M} \operatorname{Tr}\left(\overline{\mathbf{H}}_{a u_{m} j}^{H} \widehat{V}_{1}\right)+\left(\bar{L}_{b u_{m}}+\bar{L}_{b u_{m} f}\right) \widehat{V}_{2} \\
& \quad+\sigma_{m}^{2} \geq \Gamma_{m} \\
& \quad \forall m \in \mathscr{M}
\end{aligned}
$$

$$
\xi\left(\operatorname{Tr}\left(\bar{G}_{b e_{k}}^{H} \widehat{V}_{2}\right)+\operatorname{Tr}\left(\bar{G}_{b e_{k} f}^{H} \widehat{V}_{2}\right)+\sigma_{e, k}^{2}\right) \geq Q_{k}
$$

$$
\begin{gathered}
\widehat{V}_{1}(n, n)=1, \quad n=1,2, \ldots,\left(N_{1}+1\right), \\
\widehat{V}_{2}(n, n)=1, \quad n=1,2, \ldots,\left(N_{2}+1\right), \\
\operatorname{Rank}\left(\widehat{V}_{1}\right)=1, \operatorname{Rank}\left(\widehat{V}_{2}\right)=1 .
\end{gathered}
$$

Problems (20a)-(20j) are nonconvex with the nonconvex constraints (20c)-(20e) and Rank-1 constraints (20h)-(20i). Similar to (12a)-(12h), formulas (20c), (20d), and (20e) are reformulated to convex constraints by employing Taylor series expansion. Firstly, we introduce auxiliary variables $x_{2}^{\prime},\left\{x_{4, k}^{\prime}\right\}$, denote $x_{1}^{\prime}[n],\left\{x_{3, k}^{\prime}[n]\right\}$ as the value solved at the $n$th iteration, and denote $\bar{x}_{1}^{\prime}[n],\left\{x_{3, k}{ }^{\prime}[n]\right\}$ as a feasible point at the $n$th iteration. Then, $(20 \mathrm{c}),(20 \mathrm{~d})$, and (20e) can be approximated in an iterative manner:

$$
\begin{gathered}
\sum_{m} \operatorname{Tr}\left(\bar{H}_{\mathrm{aim}}^{H} \widehat{V}_{1}\right)+\operatorname{Tr}\left(\bar{G}_{\mathrm{bif}}^{H} \widehat{V}_{2}\right)+\sigma_{I}^{2} \\
\leq 2^{x_{1}^{\prime}[n]}\left[1+\left(x_{1}^{\prime}[n]-\bar{x}_{1}^{\prime}[n]\right) \ln 2\right], \\
\operatorname{Tr}\left(\bar{G}_{b i}^{H} \widehat{V}_{2}\right)+\sum_{m} \operatorname{Tr}\left(\bar{H}_{\mathrm{aim}}^{H} \widehat{V}_{1}\right)+\operatorname{Tr}\left(\bar{G}_{\mathrm{bif}}^{H} \widehat{V}_{2}\right)+\sigma_{I}^{2} \geq 2^{x_{2}^{\prime}},
\end{gathered}
$$

$$
x_{1}^{\prime}-x_{2}^{\prime} \geq \varphi^{\prime}
$$

$$
\sum_{m} \operatorname{Tr}\left(\bar{H}_{\mathrm{ame}_{k}}^{H} \widehat{V}_{1}\right)+\operatorname{Tr}\left(\bar{G}_{b e_{k} f}^{H} \widehat{V}_{1}\right)+\sigma_{e, k}^{2}+\operatorname{Tr}\left(\bar{G}_{b e_{k}}^{H} \widehat{V}_{2}\right) \leq 2^{x_{3, k \prime}}
$$

$$
\begin{gathered}
\sum_{m} \operatorname{Tr}\left(\bar{H}_{\mathrm{ame}_{k}}^{H} \widehat{V}_{1}\right)+\operatorname{Tr}\left(\bar{G}_{b e_{k} f}^{H} \widehat{V}_{1}\right)+\sigma_{e, k}^{2} \geq 2^{x_{4, k \prime}}, \\
x_{3, k}^{\prime}-x_{4, k}^{\prime} \leq u^{\prime}, \quad \forall k \in \mathscr{K} .
\end{gathered}
$$

For the constraint of Rank-1 like (20j), the classic approach is firstly dropping $(20 \mathrm{j})$ and solving problem (20a)-(20j). Then, the Gaussian randomization method is employed to find a Rank-1 solution when the optimal solution does not satisfy Rank-1. However, the solution deriving from Gaussian randomization may not satisfy all the constraints, which is not a feasible solution. Fortunately, invoked by [25], SROCR approach is suited for problems (20a)-(20j) to approximate a Rank-1 solution. First, constraint $(20 \mathrm{~g})$ is replaced with the relaxed constraint which makes the largest eigenvalue to trace ratio of $\widehat{V}_{j}$ with the 
factor $\xi_{j}^{(i)} \in[0,1]$. Then, problems $(20 \mathrm{a})-(20 \mathrm{j})$ are formulated as

$$
\begin{aligned}
& \max _{\widehat{V}_{1}, \widehat{V}_{2}} z^{\prime}, \\
& \text { s.t. } \quad \mathbf{u}_{\max }\left(\widehat{V}_{j}^{(i)}\right)^{H} \widehat{V}_{j} \mathbf{u}_{\max }\left(\widehat{V}_{j}^{(i)}\right) \geq \xi_{j}^{(i)} \operatorname{Tr}(\widehat{V}), \quad j=1,2,
\end{aligned}
$$

(20b), (20f) - (20i), (21a) - (21f),

where $\mathbf{u}_{\max }\left(\widehat{V}_{j}^{(i)}\right)$ is the corresponding eigenvector and $\widehat{V}_{j}^{(i)}$ is the obtained solution at the $i$ th iteration. It is easily seen that problems (22a)-(22c) are a convex problem, which can be solved by CVX solver. By adjusting the factor $\xi_{j}^{(i)}$ from 0 to 1 after each iteration, we can obtain a locally optimal Rank-1 solution. The detail is similar to [26], which is omitted for conciseness. In fact, simulations show that the optimal matrices $\widehat{V}_{1}$ and $\widehat{V}_{2}$ are always Rank-1 only with the SDR method.

After extracting $\widetilde{v}_{j}$ from $\widehat{V}_{j}$, due to introducing slack variable $\bar{w}$ in (4), the phase shifts at the IRS can be given by

$$
v_{j, n}=e^{j<\left(v_{j, n} / v_{j, N} N_{j+1}\right)}, \quad n=1, \ldots, N_{j} .
$$

3.3. Overall Algorithm. To summarize, the overall algorithm to solve problem (11a)-(11e) is described in Algorithm 2, where $L$ denotes the maximum number of iterations.

3.3.1. Convergence Analysis. For the $l$ th alternating iteration, denote $R\left(\mathbf{w}_{I}^{(l)}, \mathbf{w}_{m}^{(l)}, \mathbf{f}^{(l)}, \widetilde{v}_{1}^{(l)}, \widetilde{v}_{2}^{(l)}\right)$ as the objective function value, where $\left(\mathbf{w}_{I}^{(l)}, \mathbf{w}_{m}^{(l)}, \mathbf{f}^{(l)}, \widetilde{v}_{1}^{(l)}, \widetilde{v}_{2}^{(l)}\right)$ is the feasible solution. Then, for the $(l+1)$ th iteration, $\left(\mathbf{w}_{I}^{(l+1)}, \mathbf{w}_{m}^{(l+1)}, \mathbf{f}^{(l+1)}, \widetilde{v}_{1}^{(l)}, \widetilde{v}_{2}^{(l)}\right)$ is the feasible solution of problems (12a)-(12h), and $\left(\mathbf{w}_{I}^{(l+1)}, \mathbf{w}_{m}^{(l+1)}, \mathbf{f}^{(l)}, \widetilde{v}_{1}^{(l+1)}, \widetilde{v}_{2}^{(l+1)}\right)$ is the feasible solution of problem $(20 \mathrm{a})-(20 \mathrm{j})$. Then, we have

$$
\begin{aligned}
& R\left(\mathbf{w}_{I}^{(l)}, \mathbf{w}_{m}^{(l)}, \mathbf{f}^{(l)}, \widetilde{v}_{1}^{(l)}, \widetilde{v}_{2}^{(l)}\right) \\
& \quad \stackrel{(a)}{\leq} R\left(\mathbf{w}_{I}^{(l+1)}, \mathbf{w}_{m}^{(l+1)}, \mathbf{f}^{(l+1)}, \widetilde{v}_{1}^{(l)}, \widetilde{v}_{2}^{(l)}\right) \\
& \quad \stackrel{(b)}{\leq} R\left(\mathbf{w}_{I}^{(l+1)}, \mathbf{w}_{m}^{(l+1)}, \mathbf{f}^{(l+1)}, \widetilde{v}_{1}^{(l+1)}, \widetilde{v}_{2}^{(l+1)}\right),
\end{aligned}
$$

where inequality (a) holds due to the fact that SCA-based algorithm in Step 2 converges and problems (12a)-(12h) are solved optimally. Then, similarly, inequality (b) holds. This indicates that the object value given in the $(l+1)$ th iteration is not smaller than that in the $l$ th iteration. That is to say, after each iteration, the object value is nondecreasing. Furthermore, along with the QoS constraint, the secrecy performance is upper bounded, which means that it must guarantee to converge after some iterations.

3.3.2. Complexity. The main complexity of Algorithm 2 lies on Steps 2 and 3. For Step 2, the complexity for solving (19) using the interior-point method, which is denoted as $\mathcal{O}_{1}$, depends on two parts, the number of variables and the number of constraints, and is summarized in Table 1. Then, denote $I_{\text {sca }}^{1}$ as the SCA iteration numbers, and we can have the complexity of Step 2 as $I_{\mathrm{sca}}^{1} \times \mathcal{O}_{1}$. Similarly, the complexity of Step 3 is $I_{\text {srocr }} \times I_{\text {sca }}^{2} \times \mathcal{O}_{2}$, where $\mathcal{O}_{2}$ denotes the complexity for solving (22), $I_{s c a}^{2}$ denotes the SCA iteration numbers, and $I_{\text {srocr }}$ denotes the SROCR iteration numbers. Furthermore, the major complexity of Algorithm 2 is given by $I_{a o}^{1} \times\left(I_{\text {sca }}^{1} \times \mathcal{O}_{1}+I_{\text {srocr }} \times I_{\text {sca }}^{2} \times \mathcal{O}_{2}\right)$, where $I_{\text {ao }}$ denotes the alternating iteration numbers.

Remark 1. We can easily extend our framework to the scenario consisting of multiple cells and IRSs, where crosstier interference among multiple cells should be more carefully exploited to enhance secrecy performance and guarantee the QoS requirements of different users. With our proposed algorithm, this challenging problem can be solved similarly.

\section{Numerical Results}

In this section, we present numerical results to validate the performance of the proposed scheme. Simulation setups are depicted in Figure 2, where MBS, FBS, IRS-1, IRS-2, and IR are located at $(0,0),(50,50),(5,0),(50,52)$, and $(75,75)$ in meter $(\mathrm{m})$, respectively. The MBS serves 2 MUs which are located at $(0,40)$ and $(40,0)$ in meter $(\mathrm{m})$, respectively, and ERs are randomly deployed on a circle with a radius of $3 \mathrm{~m}$ centered on the FBS.

The channel between node $i$ to node $j$ is modeled as $h_{i j}=\sqrt{L_{0} d_{i j}^{-c_{i j}}} g_{i j}$, where $L_{0}=-30 \mathrm{~dB}$ is the large-scale path loss at the reference distance $d_{0}=1 \mathrm{~m}, d_{i j}$ represents the distance from $i$ to $j$, and $c_{i j}$ represents the path loss exponent. Besides, $g_{i j}$ is the small-scale fading component and is given by

$$
g_{i j}=\sqrt{\frac{\beta_{i j}}{1+\beta_{i j}}} g_{i j}^{\mathrm{LOS}}+\sqrt{\frac{1}{1+\beta_{i j}}} g_{i j}^{\mathrm{NLOS}},
$$

where $\beta_{i j}$ denotes the Rician factor, $g_{i j}^{\mathrm{NLOS}}$ is the non-lineof-sight (NLoS) component, and $g_{i j}^{\mathrm{LOS}}$ is the LoS component. We assume that for cross-layer channels, only NLoS component exists and the corresponding $\beta$ is set to 0 ; for the reflecting channel in the same cell, since IRSs are deployed vertically higher than users, where exists less scatter [15], Rician factor and path loss exponent are set as $c_{a r 1}=c_{b r 2}=2.5, \beta_{a r 1}=\beta_{b r 2}=\infty$; for the other channels, we set $c=3, \beta=10$, respectively. The rest of the parameters are listed as follows: $M_{a}=4, M_{b}=3, \xi=0.5, Q_{k}=-20 \mathrm{dBm}$, $\forall k \in \mathscr{K}, \quad \Gamma_{m}=5 \mathrm{dBm}, \quad \forall m \in \mathscr{M}, \quad \varepsilon=0.002, \quad L=20$, $\sigma_{m}^{2}=\sigma_{I}^{2}=\sigma_{e}^{2}=-60 \mathrm{dBm}$, and $q=10$. The following results are achieved by the average of simulating over 100 random channel realizations.

With the purpose of analyzing the advantage of our scheme, three benchmark schemes are also described as follows. 
Initialization: set $l=0$ and randomly construct reflection coefficients vector as $\widetilde{v} \lim _{x \longrightarrow \infty 1}{ }^{(0)}, \widetilde{v}_{2}^{(0)}$;

(1) Repeat

(2) Solve (12a)-(12h) for given $\widetilde{v}_{1}^{(l-1)}, \widetilde{v}_{2}^{(l-1)}$ according to Algorithm 1 and obtain the optimal $\mathbf{w}_{m}^{(l)}, \mathbf{w}_{I}^{(l)}, \mathbf{f}^{(l)}$;

(3) Solve (20a)-(20j) for given $\mathbf{w}_{m}^{(l)}, \mathbf{w}_{I}^{(l)}, \mathbf{f}^{(l)}$ and obtain the optimal $\widetilde{v}_{1}^{(l)}, \widetilde{v}_{2}^{(l)}$;

(4) Updata $l=l+1$;

(5) Until the decreasing rate of the objective value is below the error or the iteration number meets $l=L$.

Algorithm 2: Proposed algorithm for solving problem (11a)-(11e).

TABLE 1: Computational complexity analysis for the proposed algorithm.

\begin{tabular}{|c|c|c|c|c|c|}
\hline \multirow[b]{2}{*}{$\begin{array}{l}\text { Algorithm } \\
\text { complexity }\end{array}$} & \multicolumn{2}{|c|}{ Variables } & \multirow[b]{2}{*}{$\begin{array}{c}\text { PSD } \\
\text { constraints }\end{array}$} & \multirow[b]{2}{*}{$\begin{array}{c}\text { SOC } \\
\text { constraints }\end{array}$} & \multirow[b]{2}{*}{ Slack constraints } \\
\hline & $\begin{array}{c}\text { Design variables (size, } \\
\text { number) }\end{array}$ & $\begin{array}{c}\text { Slack variables (size, } \\
\text { number) }\end{array}$ & & & \\
\hline Step 2 & $\begin{array}{c}\left(M_{a} \times M_{a}, M\right) \\
\left(M_{b} \times M_{b}, 2\right)\end{array}$ & $(1 \times 1,5 K+2 q+11)$ & $\begin{array}{c}\left(M_{a} \times M_{a}, M\right) \\
\left(M_{b} \times M_{b}, 2\right)\end{array}$ & $(2 q+4)$ & $(M+7 K+5)$ \\
\hline Step 3 & $\begin{array}{l}\left(N_{1} \times N_{1}, 1\right) \\
\left(N_{2} \times N_{2}, 1\right)\end{array}$ & $(1 \times 1,5 K+2 q+13)$ & $\begin{array}{l}\left(N_{1} \times N_{1}, 1\right) \\
\left(N_{2} \times N_{2}, 1\right)\end{array}$ & $(2 q+4)$ & $\left(N_{1}+N_{2}+M+7 K+5\right)$ \\
\hline
\end{tabular}

Scheme 1. With IRS but without AN (IRS, no AN). In this case, FBS does not transmit AN vector by setting $\mathbf{f}=0$ and solving problems (11a)-(11e).

Scheme 2. With IRS and random phase shifts (IRS random phase, AN). In this case, the phase shift of each element at the IRS is randomly chosen in $(0,2 \pi)$. Then, we only design the transmit beamforming at the MBS or FBS by solving problems (12a)-(12h).

Scheme 3. Without IRS but with AN (No IRS, AN). In this case, the MBS or FBS serves terminals without IRSs [5], by setting $\widetilde{v}_{j}=\left[0_{N_{j} \times 1}, 1\right]$ and solving problems (12a)-(12h).

The secrecy rate at IR versus the system transmit power is shown in Figure 3. We can observe that with the increase of the transmit power, the secrecy rates of all the schemes improve, in other words, increasing the transmission power in exchange for security. Under the same power usage constraint, the proposed scheme significantly outperforms the other three benchmark schemes. It indicates that by providing more spatial DoFs, passive IRS can enhance security with less energy consumption. Moreover, as the transmission power is relatively small, the performance of the scheme without IRS but with $\mathrm{AN}$ outperforms the scheme with IRS but without AN, and as the transmission power increases to a level, the performance gap is gradually narrowing. The reason is that due to very short access distance of ER, the constraint of energy harvesting and the objective of degrading wiretapping channel are hard to meet simultaneously, and without the constraint of modulus 1 , the AN technique can provide more adjustment degree than IRS. Thus, it further illustrates that the AN technique is still needed in the power-limited system and reasonable parameters are very necessary for SWIPT-enabled HetNets.

Figure 4 shows the secrecy rate gains versus the number of ERs. We can observe that with the increase of the number of ERs, the secrecy rates of four schemes are all decreasing.

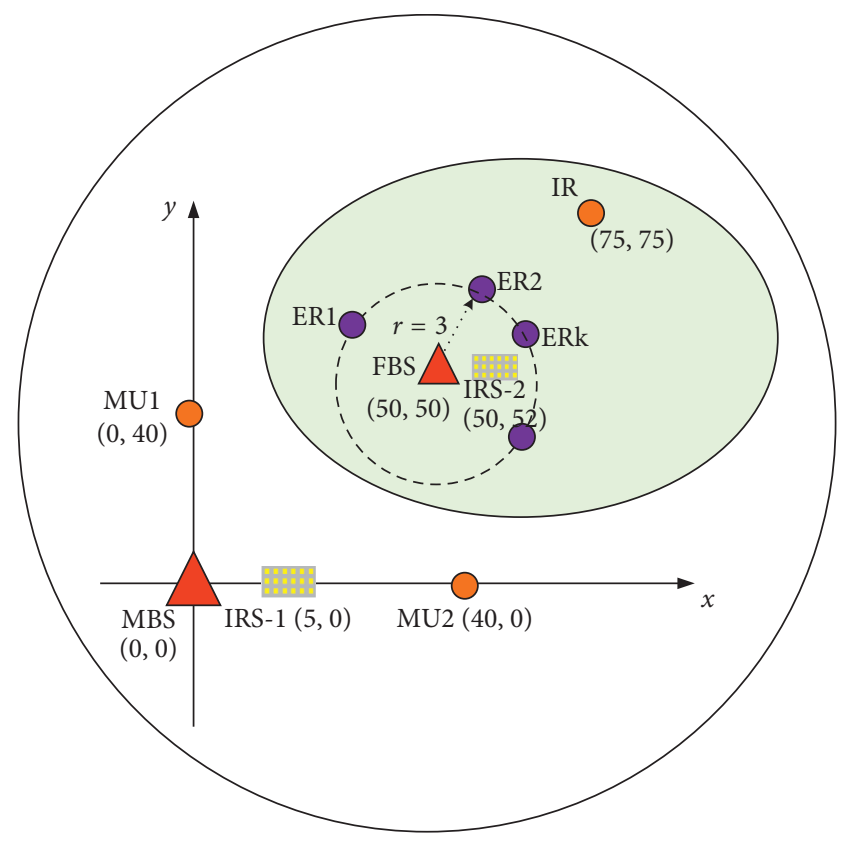

FIgURE 2: Simulation setup.

As we know, when the number of ERs exceeds that of the BS's antennas, it is hard to suppress the signal in the ERs' direction to guarantee non-negative secrecy, while for Scheme 2, only with passive IRS, the system can maintain a positive secrecy rate. Moreover, the scheme with passive IRS and AN significantly outperforms the other three benchmark schemes, which indicates that IRS can provide an effective choice for secure massive IoT scenarios.

We further examine the secrecy rate at IR versus different $N_{\text {IRS }}$ in Figure 5. It is observed that the performance of the schemes with IRS is increasing as the number of reflecting elements increases. The reason is that IRS with larger elements can provide more DoFs to degrade the reception at the ERs and satisfy QoS constraints. In fact, the 


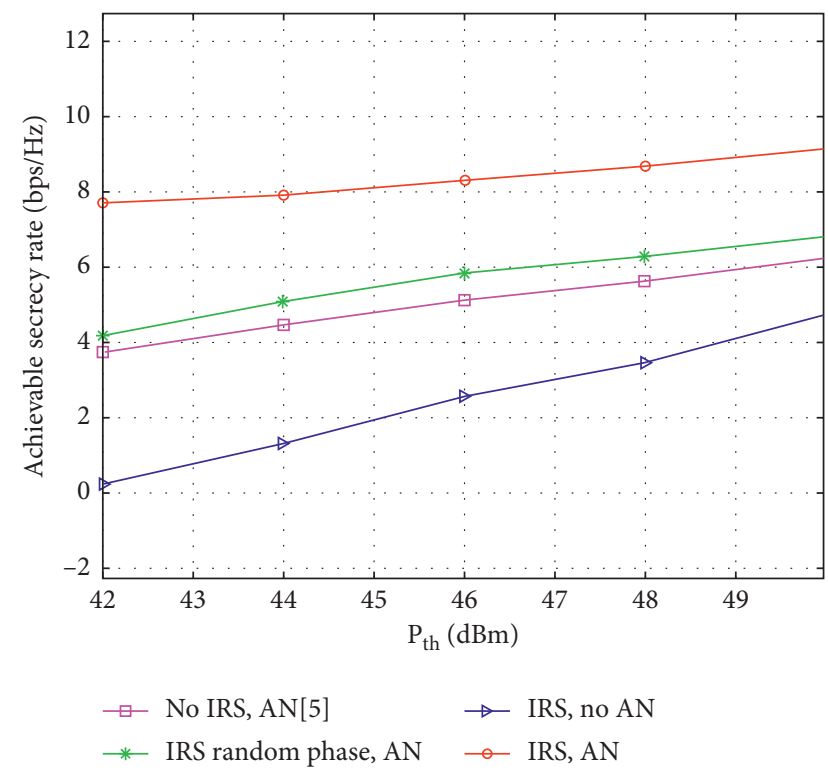

FIgUre 3: Achievable secrecy rate at IR versus the total transmit power $\left(N_{\text {IRS }}=30, K_{\mathrm{ER}}=3\right)$.

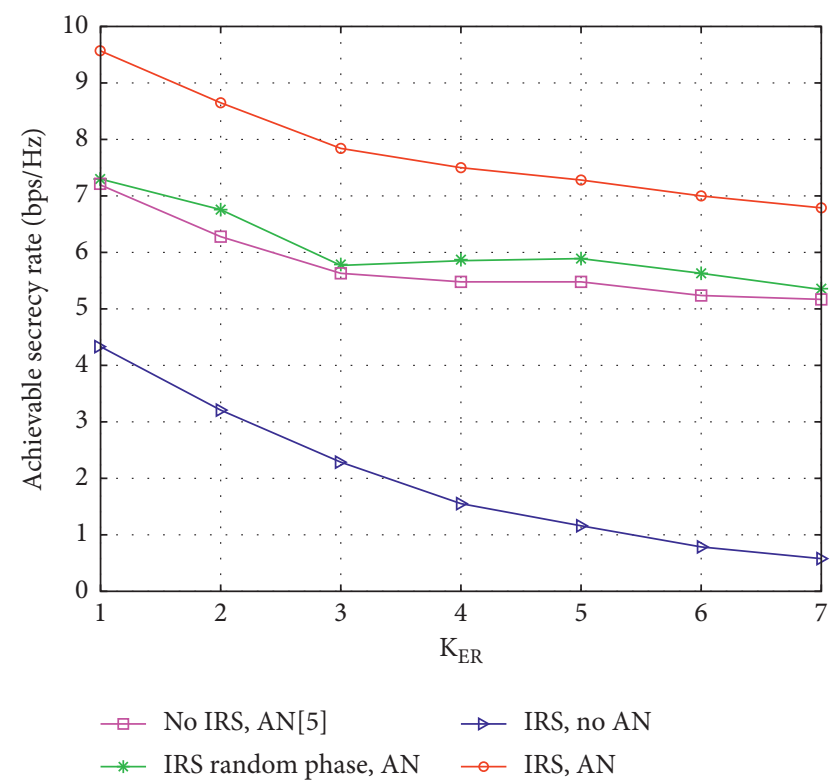

FIgURE 4: Achievable secrecy rate at IR versus the number of ERs $\left(N_{\text {IRS }}=20, P_{\text {th }}=48 \mathrm{dBm}\right)$.

number of reflecting elements can reach 100 or more at a low cost [9], which could bring more performance gain. From Figures 3-5, we can also see that the performance of Scheme 2 is slightly better than that of Scheme 3. It means that IRS, without well designed reflection coefficients, may not enhance the performance, although the number of channel paths actually increases. And the performance gap becomes more obvious with increasing number of reflecting elements.

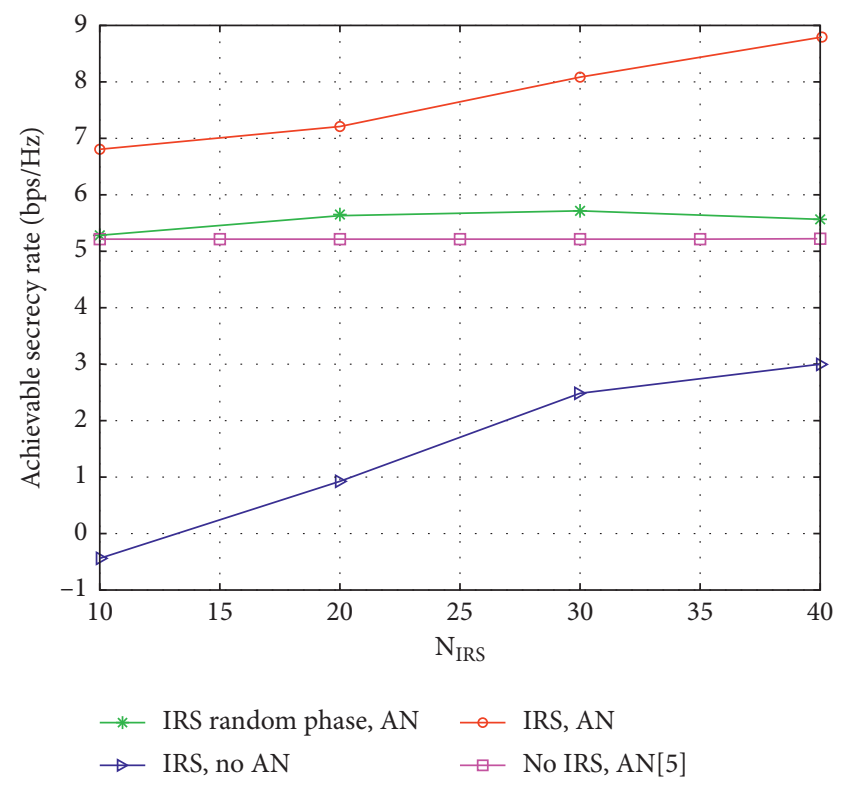

FIgURe 5: Achievable secrecy rate at IR versus different $N_{\text {IRS }}$ $\left(K_{\mathrm{ER}}=2, P_{\mathrm{th}}=45 \mathrm{dBm}\right)$.

\section{Conclusion}

In this paper, we have presented an investigation of IRS-assisted secure SWIPT HetNet system. The secrecy rate maximization problem under the constraint of QoS is established by jointly designing transmit beamforming vectors, AN vectors, and phase shifts. Specifically, we invoke an alternating optimization and SCA techniques to transform the nonconvex original problem into convex and obtain a suboptimal solution. $\mathrm{Nu}-$ merical results show that the proposed scheme could effectively exploit co-channel interference as a favorable factor, and it could maintain promising security performance when the number of terminals increases. Furthermore, considering the passive nature of the IRS, the instantaneous CSI of the reflecting link is difficult to obtain. Therefore, it is worthwhile to investigate the resource allocation scheme under outdated CSI in the future.

\section{Appendix}

\section{A. Proof of Proposition 1}

The original problem (12a)-(12h) can be equivalently written as

$$
\begin{gathered}
\max t_{0}, \\
\text { s.t. } \quad(12 \mathrm{f}),(12 \mathrm{~g}),(12 \mathrm{~h}),(12 \mathrm{i}), \frac{1}{2}, \\
\operatorname{Tr}\left(\widehat{G}_{b i}^{H} \mathbf{W}_{I}\right) \geq\left(t_{1}-1\right)\left(\sum_{m} \operatorname{Tr}\left(\widehat{H}_{a i}^{H} \mathbf{W}_{m}\right)+\operatorname{Tr}\left(\widehat{G}_{b i}^{H} \mathbf{F}\right)+\sigma_{I}^{2}\right),
\end{gathered}
$$




$$
\begin{aligned}
\operatorname{Tr}\left(\widehat{G}_{b e_{k}}^{H} \mathbf{W}_{I}\right) \leq & \left.\frac{1}{t_{2}-1}\right) \\
& \left(\sum_{m} \operatorname{Tr}\left(\widehat{H}_{a e_{k}}^{H} \mathbf{W}_{m}\right)+\operatorname{Tr}\left(\widehat{G}_{b e_{k}}^{H} \mathbf{F}\right)+\sigma_{e, k}^{2}\right),
\end{aligned}
$$

$\forall k \in \mathscr{K}$,

$$
t_{0} \leq t_{1} t_{2}
$$

where $t_{0}, t_{1}$, and $t_{2}$ are the introduced slack variables. Utilizing the Lagrange multiplier, the above problem can be transformed as

$$
\begin{aligned}
& L\left(\mathbf{W}_{I}, \mathbf{W}_{m}, \mathbf{F}, a, b, c, d, e\right) \\
& =t_{1} t_{2}-a\left(\operatorname{Tr}\left(\widehat{G}_{b i}^{H} \mathbf{W}_{I}\right)-\left(t_{1}-1\right)\left(\sum_{m} \operatorname{Tr}\left(\widehat{H}_{a i}^{H} \mathbf{W}_{m}\right)\right)\right. \\
& \quad-\left(t_{1}-1\right) \operatorname{Tr}\left(\widehat{G}_{b i}^{H} \mathbf{F}\right)+\sigma_{I}^{2}+b\left(\frac{1}{t_{2}-1}\right) \sum_{k} \operatorname{Tr}\left(\widehat{G}_{b e_{k}}^{H} \mathbf{W}_{I}\right) \\
& \quad+b\left(\frac{1}{t_{2}-1}\right) \sum_{k}\left(\operatorname{Tr}\left(\widehat{G}_{b e_{k}}^{H} \mathbf{F}\right)+\sigma_{e, k}^{2}-\sum_{m} \operatorname{Tr}\left(\widehat{H}_{a e_{k}}^{H} \mathbf{W}_{m}\right)\right) \\
& \quad-c \sum_{m} \Gamma_{m}\left(\sum_{i=1, i \neq m}^{M} \operatorname{Tr}\left(\widehat{H}_{a u_{m}}^{H} \mathbf{W}_{i}\right)+\widehat{L}_{b u_{m}}^{H}\left(\mathbf{W}_{I}+\mathbf{F}\right)+\sigma_{m}^{2}\right) \\
& \quad-c \sum_{m}\left(\operatorname{Tr}\left(\widehat{H}_{a u_{m}}^{H} \mathbf{W}_{m}\right)\right)-d \sum_{k} \xi\left(\operatorname{Tr}\left(\widehat{G}_{b e_{k}}^{H} \mathbf{W}_{I}\right)\right. \\
& \left.\quad-d \sum_{k}\left(\operatorname{Tr}\left(\widehat{G}_{b e_{k}}^{H} \mathbf{F}\right)+\sigma_{e, k}^{2}\right)-Q_{k}\right) \\
& \quad+e\left(\sum_{m=1}^{M} \operatorname{Tr}\left(\mathbf{W}_{m}\right)+\operatorname{Tr}\left(\mathbf{W}_{I}\right)+\operatorname{Tr}(\mathbf{F})-P_{\mathrm{th}}\right)
\end{aligned}
$$

where $a \geq 0, b \geq 0, c \geq 0, d \geq 0$, and $e \geq 0$ represent the Lagrange multipliers of problem (A.1) corresponding to the constraints in (A.1a)-(A.1e), respectively. By further simplifying, we can get

$$
\begin{aligned}
L & =\operatorname{Tr}\left(\mathbf{D}_{I} \mathbf{W}_{I}\right)+\operatorname{Tr}\left(\mathbf{D}_{m} \mathbf{W}_{m}\right)+\operatorname{Tr}\left(\mathbf{D}_{F} \mathbf{F}\right)+C, \\
\mathbf{D}_{I} & =-a \widehat{G}_{b i}^{H}+\left(b\left(\frac{1}{t_{2}-1}\right)-\mathrm{d} \xi\right) \sum_{k}\left(\widehat{G}_{b e_{k}}^{H}\right)+c \sum_{m} \Gamma_{m} \widehat{L}_{b u_{m}}^{H}+e \mathbf{I},
\end{aligned}
$$

$$
\begin{aligned}
\mathbf{D}_{m}= & a\left(\left(t_{1}-1\right)\left(\operatorname{Tr}\left(\widehat{H}_{a j}^{H}\right)-b\left(\frac{1}{t_{2}-1}\right)\right) \sum_{k} \operatorname{Tr}\left(\bar{H}_{a e_{k}}^{H}\right)\right. \\
& -c \operatorname{Tr}\left(\widehat{H}_{a u_{j}}^{H}\right)+c \sum_{i=1, i \neq j}^{M} \Gamma_{i} \operatorname{Tr}\left(\widehat{H}_{a u_{i}}^{H}\right)+e I
\end{aligned}
$$

$$
\begin{aligned}
C= & t_{1} t_{2}-\left(t_{1}-1\right) \sigma_{I}^{2}+b\left(\left(\frac{1}{t_{2}-1}\right) \sigma_{e, k}^{2}\right. \\
& -c \Gamma_{m} \sigma_{m}^{2}+d Q_{k}-e P_{\text {th }} .
\end{aligned}
$$

According to the definition of the secrecy rate and formula (11a), we can easily have $t_{1}-1 \geq 0$ and $\left(1 / t_{2}\right) \geq 0$, and $\operatorname{Rank}\left(\widehat{G}_{b i}^{H}\right) \leq 1$, and based on the properties of positive semidefinite matrix, we can get $\operatorname{Rank}\left((b)\left(1 / t_{2}-\right.\right.$ 1) $\left.-\mathrm{d} \xi) \sum_{k}\left(\widehat{G}_{b e_{k}}^{H}\right)+c \sum_{m} \Gamma_{m} \widehat{L}_{b u_{m}}^{H}+e \mathbf{I}\right)=M_{b}$. Then, we can derive the following solution: $\operatorname{Rank}\left(\mathbf{D}_{I}\right) \geq M_{b}-1$. Further, according to Karush-Kuhn-Tucker condition, we get $D_{I} \mathbf{W}_{I}=0$.

Therefore, we can get $\operatorname{Rank}\left(\mathbf{W}_{I}\right) \leq 1$. When $\operatorname{Rank}\left(\mathbf{W}_{I}\right)=0$, the transmission rate of IR is zero, which is unfeasible in this case. Therefore, the optimal beamforming $\mathbf{W}_{I}$ must be Rank-1. Likewise, the rank of $\mathbf{F}$ can be proved to be one, which is omitted here.

\section{Data Availability}

All figures were drawn by the tools of Visio and Matlab. The data used to support this study are available from the corresponding author upon request.

\section{Conflicts of Interest}

The authors declare that there are no conflicts of interest regarding the publication of this paper.

\section{Acknowledgments}

This study was supported in part by the National Natural Science Foundation of China under grant nos. 61871404, 61801435, and 61941114 and in part by the China Postdoctoral Science Foundation under grant no. 2018M633733.

\section{References}

[1] S. Akbar, Y. Deng, A. Nallanathan, M. Elkashlan, and A.-H. Aghvami, "Simultaneous wireless information and power transfer in \$K\$ -tier heterogeneous cellular networks," IEEE Transactions on Wireless Communications, vol. 15, no. 8, pp. 5804-5818, 2016.

[2] J. Tang, A. Shojaeifard, D. K. C. So, K.-K. Wong, and N. Zhao, "Energy efficiency optimization for comp-swipt heterogeneous networks," IEEE Transactions on Communications, vol. 66 , no. 12 , pp. 6368-6383, 2018. 
[3] Q. Shi, W. Xu, J. Wu, E. Song, and Y. Wang, "Secure beamforming for mimo broadcasting with wireless information and power transfer," IEEE Transactions on Wireless Communications, vol. 14, no. 5, pp. 2841-2853, 2015.

[4] J. M. Hamamreh, H. M. Furqan, and H. Arslan, "Classifications and applications of physical layer security techniques for confidentiality: a comprehensive survey," IEEE Communications Surveys \& Tutorials, vol. 21, no. 2, pp. 1773-1828, 2019.

[5] B. Li, Z. Fei, and Z. Chu, "Optimal transmit beamforming for secure SWIPT in a two-tier HetNet," IEEE Communications Letters, vol. 21, no. 11, pp. 2476-2479, 2017.

[6] H. Ma, J. Cheng, and X. Wang, "Proportional fair secrecy beamforming for miso heterogeneous cellular networks with wireless information and power transfer," IEEE Transactions on Communications, vol. 67, no. 8, pp. 5659-5673, 2019.

[7] B. Zhang, K. Huang, L. Jin, and M. Yi, "Robust secrecy energy efficiency optimization in heterogeneous networks with swipt: centralized and distributed design," IET Communications, vol. 13, no. 17, pp. 2857-2870, 2019.

[8] T. J. Cui, M. Q. Qi, X. Wan, J. Zhao, and Q. Cheng, "Coding metamaterials, digital metamaterials and programmable metamaterials," Light: Science \& Applications, vol. 3, no. 10, p. e218, 2014.

[9] Q. Wu and R. Zhang, "Towards smart and reconfigurable environment: intelligent reflecting surface aided wireless network," IEEE Communications Magazine, vol. 58, no. 1, pp. 106-112, 2019.

[10] M. Cui, G. Zhang, and R. Zhang, "Secure wireless communication via intelligent reflecting surface," IEEE Wireless Communications Letters, vol. 8, no. 5, pp. 1410-1414, 2019.

[11] X. Lu, W. Yang, X. Guan, Q. Wu, and Y. Cai, "Robust and secure beamforming for intelligent reflecting surface aided mmwave miso systems," IEEE Wireless Communication Letters, vol. 9, no. 12, pp. 2068-2072, 2020.

[12] H. Guo, Y.-C. Liang, J. Chen, and E. G. Larsson, "Weighted sum-rate maximization for intelligent reflecting surface enhanced wireless networks," in Proceedings of the 2019 IEEE Global Communications Conference (GLOBECOM), pp. 1-6, Waikoloa, HI, USA, December 2019.

[13] C. Huang, A. Zappone, G. C. Alexandropoulos, M. Debbah, and C. Yuen, "Reconfigurable intelligent surfaces for energy efficiency in wireless communication," IEEE Transactions on Wireless Communications, vol. 18, no. 8, pp. 4157-4170, 2019.

[14] W. Jiang, Y. Zhang, J. Wu, W. Feng, and Y. Jin, "Intelligent reflecting surface assisted secure wireless communications with multiple-transmit and multiple-receive antennas," IEEE Access, vol. 8, pp. 86659-86673, 2020.

[15] Q. Wu and R. Zhang, "Joint active and passive beamforming optimization for intelligent reflecting surface assisted swipt under QOS constraints," IEEE Journal on Selected Areas in Communications, vol. 38, no. 8, pp. 1735-1748, 2020.

[16] W. Shi, X. Zhou, L. Jia, Y. Wu, F. Shu, and J. Wang, "Enhanced secure wireless information and power transfer via intelligent reflecting surface," IEEE Communications Letters, p. 1. In press, 2020.

[17] L. Liu, R. Zhang, and K.-C. Chua, "Secrecy wireless information and power transfer with miso beamforming," IEEE Transactions on Signal Processing, vol. 62, no. 7, pp. 1850-1863, 2014.

[18] Q. Wu, S. Zhang, B. Zheng, C. You, and R. Zhang, "Intelligent reflecting surface aided wireless communications: a tutorial," 2020, https://arxiv.org/abs/2007.02759.

[19] M. Sheng, L. Wang, X. Wang, Y. Zhang, C. Xu, and J. Li, "Energy efficient beamforming in miso heterogeneous cellular networks with wireless information and power transfer," IEEE Journal on Selected Areas in Communications, vol. 34, no. 4, pp. 954-968, 2016.

[20] H. Zhang, Y. Huang, C. Li, and L. Yang, "Secure beamforming design for swipt in miso broadcast channel with confidential messages and external eavesdroppers," IEEE Transactions on Wireless Communications, vol. 15, no. 11, pp. 7807-7819, 2016.

[21] Z. Zhu, Z. Chu, N. Wang, S. Huang, Z. Wang, and I. Lee, "Beamforming and power splitting designs for an-aided secure multi-user mimo swipt systems," IEEE Transactions on Information Forensics and Security, vol. 12, no. 12, pp. 2861-2874, 2017.

[22] D.-H. Chen, Y.-C. He, X. Lin, and R. Zhao, "Both worst-case and chance-constrained robust secure swipt in miso interference channels," IEEE Transactions on Information Forensics and Security, vol. 13, no. 2, pp. 306-317, 2018.

[23] K.-G. Nguyen, L.-N. Tran, O. Tervo, Q.-D. Vu, and M. Juntti, "Achieving energy efficiency fairness in multicell miso downlink," IEEE Communications Letters, vol. 19, no. 8, pp. 1426-1429, 2015.

[24] X. Guan, Q. Wu, and R. Zhang, "Intelligent reflecting surface assisted secrecy communication: is artificial noise helpful or not?" IEEE Wireless Communications Letters, vol. 9, no. 6, pp. 778-782, 2020.

[25] P. Cao, J. Thompson, and H. V. Poor, "A sequential constraint relaxation algorithm for rank-one constrained problems," in Proceedings of the 2017 25th European Signal Processing Conference (EUSIPCO), pp. 1060-1064, Kos, Greece, September 2017.

[26] X. Mu, Y. Liu, L. Guo, J. Lin, and N. Al-Dhahir, "Exploiting intelligent reflecting surfaces in noma networks: joint beamforming optimization," IEEE Transactions on Wireless Communications, vol. 19, no. 10, pp. 6884-6898, 2020. 